\title{
Single Stage Lateral Based Flap Urethroplasty and Two Stage Urethroplasty in Proximal Penile Hypospadias with Chordee: A Prospective Case Control Study
}

Ipsita Biswas ${ }^{1+}$

Abhi Kumar Chakraborty ${ }^{2}$

Kazi Md. Noor-ul Ferdous

Abdul Aziz'

Hafiza Sultana ${ }^{3}$

'Department of Pediatric Surgery Dhaka Shishu (Children) Hospital Dhaka, Bangladesh.

${ }^{2}$ Department of Pediatric Surgery Shaheed Suhrawardy Medical College \& Hospital Dhaka, Bangladesh.

${ }^{3} \mathrm{NIPSOM}$

Dhaka, Bangladesh.

\begin{abstract}
Aims: Hypospadias is one of the commonest congenital abnormalities of the male genitalia. Proximal penile hypospadias is the challenging condition to correct Lateral based flap (LB) procedure combines meatal - based flap and pedicle flap technique into one procedure without the need for an intervening anastomosis. In this study we compared Lateral based flap (LB) procedure with conventional two stage urethroplasty for proximal penile hypospadias with chordee. The aim of this study was to find out the outcome of lateral based flap urethroplasty in treating proximal penile hypospadias with chordee over two stage procedure. Study Design: In this prospective study that carried out from $1^{\text {st }}$ January 2012 to $30^{\text {th }}$ June 2013 , patients with proximal penile hypospadias from 6 months to 144 months (12yrs) of age attending at Dhaka Shishu (Children) Hospital out patient department were primarily enrolled for this study. Patients were grouped into A and B on the basis of repair by Lateral based flap single stage urethroplasty and by two stage techniques respectively. Results were evaluated after 6 months of urethroplasty and onward. Results: Total 24 Patients, ages ranged from 7 months to 10 years (mean 3.97 years) of proximal penile hypospadias (12patients in each group) were included. For Lateral based flap (LB) procedure (Group-A), mean time required for urethroplasty was 3.23 hours. On the other hand, for control group (Group-B) mean total time required was 3.99 hours (for orthoplasty 1.67 hours and for urethroplasty 2.32 hours). Urethrocutaneous fistula developed in $16.6 \%$ (2 patients) and glanular dehiscence in $8.3 \%$ ( 1 patient) in each group. In addition 25\% (3 patients) in control group developed meatal stenosis. Satisfactory results were observed more than $83 \%$ of group-A patients and $58.3 \%$ of group B patients. Conclusions: The study reflects that lateral based flap (LB) procedure technique may offer the patients with proximal and severe forms of hypospadias, a good chance of a single stage operation with high success rate.
\end{abstract}

Key words: Lateral based flap; Proximal penile hypospadias; Chordee.

\section{INTRODUCTION}

Hypospadias is one of the commonest congenital abnormalities of the male occurring in approximately 1 in 300 male children. Proximal variety constitutes about $20 \%$ of all hypospadias and proximal penile hypospadias constitute about $15 \%$ of proximal variety ${ }^{1}$.

The challenge of hypospadias repair has stimulated surgeons for centuries. As a result more than 300 types of urethroplasty for repair have been explored in depth and in great detail. Proximal penile hypospadias is one of the most challenging conditions to correct. The multiple numbers of procedures that has been described over the years is indicative of the fact that no procedure has been universally acceptable or successful. Many have chosen to perform staged procedure because this has the advantage that the varied anatomical issues can be fixed sequentially with different aspects of the problem being tackled in time. A disadvantage of this approach is that the patient needs to undergo at least two and often more procedures ${ }^{2}$

\section{Dr. Ipsita Biswas}

Department of Pediatric Surgery

Bangladesh Institute of Child Health

Dhaka Shishu (Children) Hospital

Dhaka, Bangladesh.

Mobile: +8801711884609

Email: ipsitabiswas74@gmail.com 
The standard two staged approach involve initial correction of penile curvature along the preparation of a ventral bed of tissue. This neourethral plate can then be tubularized at a second setting.

One stage procedures are undoubtedly attractive, desirable and popular. They are associated with a shorter hospital stay and are more convenient for both patient and surgeon alike ${ }^{3}$.

Repair of proximal hypospadias in one stage is among the most difficult challenges reconstructive surgeon may face. In attempt to solve this dilemma for patients with severe hypospadias, a repair with dual blood supply will offer many advantages. With this concept in mind, the lateral based flap (LB) repair was conceived. This procedure combines meatal -based flap and pedicle flap technique into one procedure without the need for an intervening anastomosis. It also allows for extensive excision of ventral chordee and urethral plate without damaging the flap ${ }^{4}$.

For proximal hypospadias single stage urethroplasty with lateral based flap procedure have observed encouraging outcome in various studies ${ }^{3}$.

The present study compared two methods with the hope of better outcome with lateral based flap single stage urethroplasty for proximal penile hypospadias with chordee.

\section{MATERIALS \& METHODS}

A prospective comparative study was performed in the Department of pediatric surgery, Dhaka Shishu (Children) Hospital, Dhaka from $1^{\text {st }}$ January 2012 to $30^{\text {th }}$ June, 2013 for a total period of 18 months. All patients from 6 months to 12 years of age with proximal penile hypospadias with chordee, not treated previously were target group. After proper counseling and written informed consent from the guardians all patients were divided randomly by lottery into group A, who were subjected to repair by lateral based flap single stage urethroplasty and group B, subjected to repair by two stage techniques-as a control. Preoperative evaluation including detailed history with background information, physical examination and laboratory investigations were performed.

Surgical Technique: All patients were operated under general anesthesia with caudal block. Urinary diversion was provided by means of placing a feeding tube (6 Fr, $7 \mathrm{Fr}, 8 \mathrm{Fr}, 10 \mathrm{Fr}$ etc according to the age of the patient) in the neourethra and fixed with the glans.

In LBF procedure a Y shaped incision is outlined on the glans. The center of the $\mathrm{Y}$ is at the tip of the glans and where the tip of the neo-meatus will be located. The Y-shaped incision is deepened down to the corpora, and these results in 3 flaps, 1 small (median) flap and 2 large lateral glanular wings. The 3 flaps are elevated creating a space for the neo urethra. Any fibrous bands or chordee tissue is meticulously excised. A rectangular skin strip is outlined extending proximally from the urethral meatus to the tip of the glans. This strip is taken from the lateral penile skin and extends into the outer preputial skin. Several interrupted stitches assist in orientation, and the urethroplasty is carried out from proximal to distal in a subcuticular continuous manner using $6 / 0$ vicryl on a cutting needle (Fig 1). The neo-meatus is constructed by suturing the terminal end of the neo-urethra to the central $\mathrm{V}$ of the glans.
Tunica vaginalis was used to provide a complete covering for the neo-urethra (Fig 2). The wings of the glans are wrapped around the neourethra and sutured together in the midline.

Ointment and compression dressing is applied for 5days, thereafter the wound is left exposed.

In two stage procedure the orthoplasty was done in first stage. Second stage was performed 6 months after $1^{\text {st }}$ operation. In second stage a $15 \mathrm{~mm}$ diameter strip is measured extending to the tip of the glans. The strip is tubularized with a running subcuticular stitch with $6 / 0$ vicryl up to the tip of the glans. The lateral skin edges are mobilized and the remaining tissue is closed over the repair in at least two layers.

Postoperatively all patients were treated by antibiotics and analgesic. Second-generation intravenous cephalosporin was given postoperatively for 2 weeks. After urethroplasty (for both groups) urinary diversion in the form of urethral catheter were kept in position for 10 days. Findings were registered in each follow up at 10 th day (day of discharge), $3^{\text {rd }}$ week, $5^{\text {th }}$ week, $8^{\text {th }}$ week, $12^{\text {th }}$ week postoperatively. Results were evaluated after 6 months and onward.

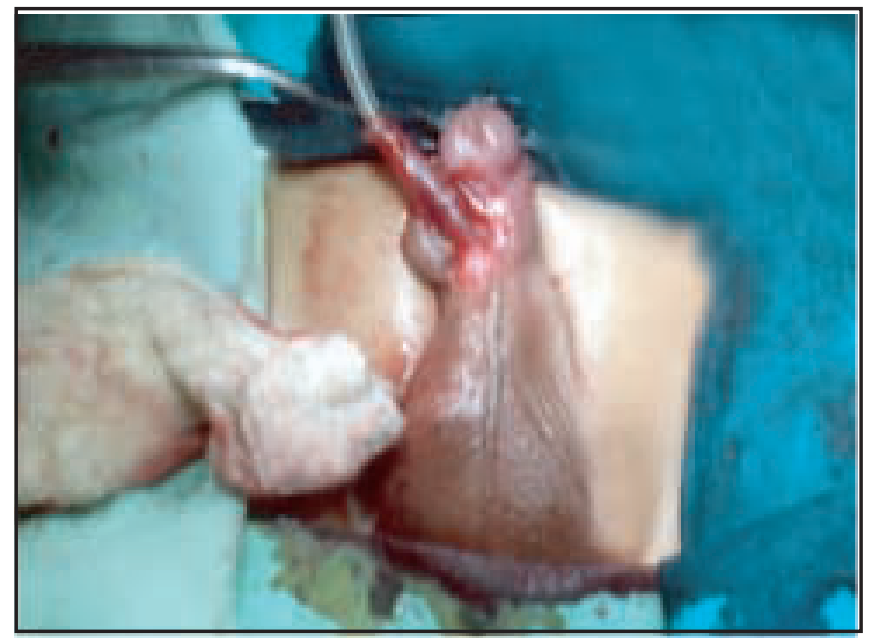

Figure 1 : After tubularization of lateral based flap

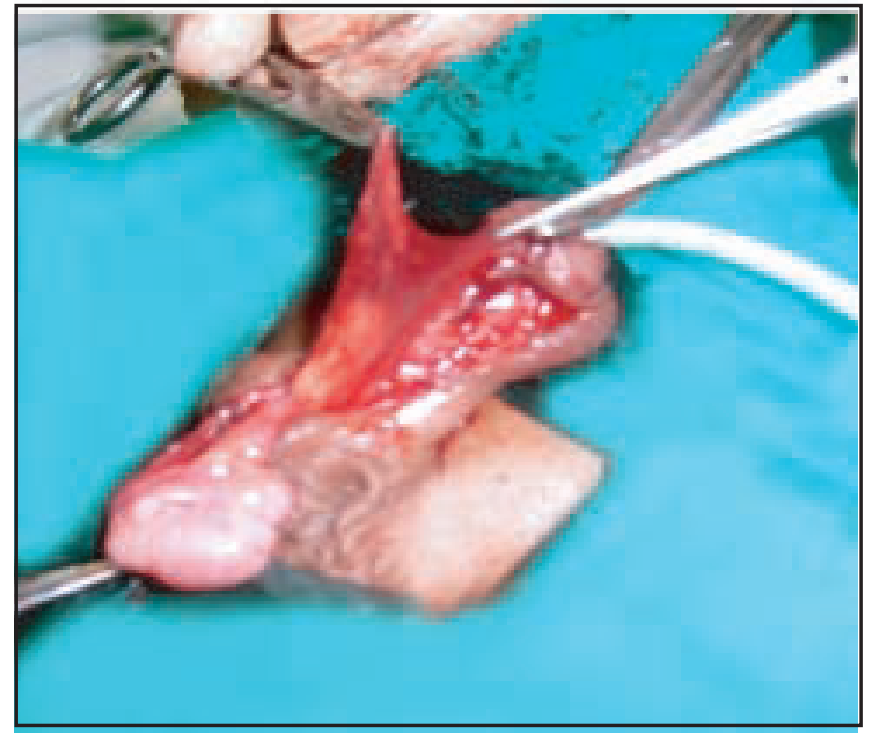

Figure 2: Intermediate layer by tunica vaginalis flap 


\section{RESULTS}

Total 24 patients were included in this study. 12 of them were in Group -A and 12 were in Group-B. The age of the patients at presentation for Group-A were 7 months to 10 years (mean age $3.97 \mathrm{yrs}$ ). For Group B age ranges at orthoplasty was 1 year to 8 years (mean age $3.97 \mathrm{yrs}$ ) and then urethroplasty done after 6 months. For Group A mean time required for urethroplasty was 3.23 hours and for Group B mean total time required for two stage procedure were 3.99 hours ( for orthroplasty 1.67 hours and for urethroplasty 2.32 hours) (Figure 3 ).

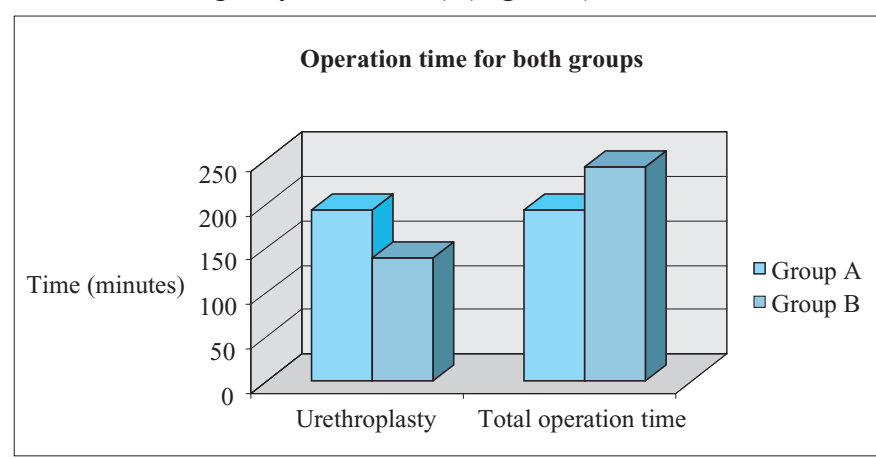

Figure 3 : Operation time for two groups

Hospital stay for group-A patients ranged from 16 days to 23 days (mean 18.67 days). For Group B hospital stay ranged from 8 days to 10 days (mean 9 days) for orthoplasty and for urethroplasty hospital stay ranged from 16 days to 21 days (mean 18.67 days).

Postoperative function and cosmetic evaluation revealed that, in Group-A $83.3 \%$ and in group B 58.3\% had no complications and achieved satisfactory result.

Results were considered satisfactory when the boy achieves a glanular meatus, single forward stream, unimpeded voiding, good cosmesis and no need for secondary surgery for the urethra.

Post operative complications developed in 2 patients in GroupA and 5 patients in Group-B. Complications were enlisted on Table-1.

Table 1 : Postoperative complications of two groups

\begin{tabular}{lrrrr} 
Infection & 0 & & 1 & $(8.3 \%)$ \\
Glanular dehiscence & 1 & $(8.3 \%)$ & 1 & $(8.3 \%)$ \\
U-C Fistula & 2 & $(16.6 \%)$ & 2 & $(16.6 \%)$ \\
Meatal stenosis & 0 & & 3 & $(25 \%)$ \\
\hline
\end{tabular}

Few children developed more than one complication

\section{DISCUSSION}

Proximal types of hypospadias have always constituted a major challenge, they are very difficult to treat, more amenable for complications and have a higher incidence of failure. Many techniques have been described for hypospadias repair. However, the surgeon has to be extremely selective when choosing the appropriate technique for his operation. The main goal should be to gain normal micturition function to construct a normal looking penis for coitus and to provide an acceptable cosmetic appearance ${ }^{5}$. One stage procedure is undoubtedly attractive, desirable and popular. They are associated with a shorter hospital stay and more convenient for both patients and surgeons alike. The preferred single stage techniques for proximal hypospadias repair are tubularized incised plate urethroplasty, transverse island tube flap. Other current techniques include the Thiersch-Duplay principle, the Onlay island flap and Lateral based flap? ${ }^{3}$.

Most of the patients with proximal hypospadias have significant chordee, which required excision of the urethral plate. So this meant that tubularized incised plate urethroplasty, Onlay island flap and Thiersch-Duplay principle are not ideal alternatives. The LB flap offered a single stage repair that is suitable when excision of the midline chordee tissue is preferred ${ }^{6}$.

It is well known that the key to ensure successful hypospadias repair with minimal complication rate is to ensure good blood supply to the flap used for urethroplasty. This was achieved designing a lateral meatal based skin flap which enjoy a dual blood supply coming from the preputial vascular pedicle and para-meatal tissue and achieved excellent outcome in one stage urethroplasty for proximal hypospadias ${ }^{4-6}$.

In our study, hypospadias constituted $2.42 \%$ of total pediatric surgical admission and proximal penile variety was $22.2 \%$ of admitted hypospadias patients. The age of the patients in this study at presentation for Group-A were 7 months to 10 years for Group B age ranges at orthoplasty was 1 year to 8 years and time interval between orthoplasty and urethroplasty were 6 months. Hadidi (2009) observed age of the patients between 1year-5 years ${ }^{6}$. In our study population, this late arrival at hospital was due to parent's ignorance, illiteracy, financial problem and referral delay.

We followed the principles of plastic surgery regarding fine instruments and fine suture materials along with precise and delicate tissue handling. In this study mean operating time for urethroplasty (3.23 hrs) for Group-A, was higher than the mean operating time for orthoplasty $(1.67 \mathrm{hrs})$ in group-B. But the mean total operation time (3.99 hrs) (for orthoplasty + urethroplasty) was more than Group-A. Similarly hospital stays for group B to complete both operations higher in comparison to single stage urethroplasty. This finding is similar to the study done by Samuel and Capps in $2002^{7}$.

Complications developed within 2 months of operation. Results were considered satisfactory when the boy achieved a glanular meatus, single forward stream, unimpeded voiding, good cosmesis and no need for secondary surgery for the urethra.

In this series complication is less in patients treated with lateral based flap urethroplasty. Satisfactory result, that is, these boys can stand to void and have a good caliber straight single stream of urine in a forward direction and cosmetic appearance of a natural vertical slit meatus situated at normal position on the glans was achieved. This finding well matched with the findings of other study ${ }^{4}$.

$25 \%$ patients of Group -B, treated by staged procedure developed meatal stenosis but none of Group-A, treated by lateral based flap procedure developed meatal stenosis. Among the three patients with meatal stenosis in Group-B, one subsequently developed fistula. 
In Lateral based flap procedure, the use of Y-V glanuloplasty and creation of a longer suture anastomotic connection between the urethral meatus and glans results a large apical meatus. Also the excision of small $\mathrm{V}$ from the apex of the neo meatus helps to achieve a terminal slit like meatus. This decreases the risk of postoperative meatal stenosis and that is usually a major contributing factor for anastomotic failure or repair break down ${ }^{6}$. In this study Urethrocutaneous fistula developed in $16.6 \%$ patients in Group-A. Hadidi in 2003 observed 10.2\% urethrocutaneous fistula in single stage LBF technique ${ }^{4}$. In group-B $16.6 \%$ patients also developed Urethrocutaneous fistula and Kuyumcuoglu et al in 2009, observed $11.8 \%$ urethrocutaneous fistula in two step urothroplasty ${ }^{5}$. 8.3\% patients of each group in our study developed glanular dehiscence. But urethroplasty was intact in both the groups. The mean follow up period was 1year 7 months (ranges from 1year to 2 years 4 months) for both the groups.
Complications are common after hypospadias surgery. In our study the urethrocutaneous fistula rate after lateral based flap technique is much less (only 16.6\%) than others studies ${ }^{4-5}$.

By comparing the surgical outcome of proximal penile hypospadias management, the lateral based flap have better outcome over the two stage procedure. So it will be more acceptable technique. Two staged procedure needs longer hospital stay, more surgical intervention that not only affects the family financially but also causes psychological trauma on parents, so two stage procedure is less convenient for both patients and surgeons.

\section{CONCLUSION}

In proximal hypospadias, single stage lateral based flap urethroplasty has higher success rate with satisfactory functional and cosmetic outcome over two stage procedures.

\section{DISCLOSURE}

All the authors declared no competing interest.

\section{REFERENCES}

1. Murphy JP. Hypospadias, In : pediatric surgery, Ashcraft KW, Marphy P, Sharp RJ, Sigalet DL \& Sanyder CL(eds), $3^{\text {rd }}$ ed, Philadelphia : W.B. Saunders company. 2000; 763-782.

2. Jayanthi VR. The modified koyanagi hypospadias repair for the one-stage repair of proximal hypospadias. Indian J Urol. 2008; 24: 206-209.

3. Hadidi A T. Single stage versus two stage repair, In : Hypospadias Surgery-An illustrated guide, Hadidi AT \& Azmi AF,(eds), Springer : Verlag Berlin Heidelberg. 2004; 261-267.

4. Hadidi AT. Lateral based flap with dual blood supply: A single stage repair for proximal hypospadias. Egypt J Reconstr Surg. 2003; 27(2): 217-223.

5. Kuyumcuoglu U, Bryildirim B, Faydaci G, TarhanF, Ozgul A. The comperision of belt- Fuqua and TIPU techniques in proximal penile hypospadias cases: retrospective analysis of 65 cases. Int Urol Nephrol, published online: 12 March 2009.

6. Hadidi AT. Lateral -based flap: a single stage urethral reconstruction for proximal hypospadias. J Pediatr Surg. 2009; 44: 797-801.

7. Sauel M, Capps S. Staged proximal hypospadias repair: Modified Thiersch duplay with midline incision in urethral plate followed by Mathieu urethroplasty. J Pediatr Surg. 2002; 37: 104-107. 